\title{
An Ex-situ Tracking Technique for Morphological Transformation in Triblock Copolymer Films
}

\author{
Yongzhi Cao, a, Wendi Tu" ${ }^{1, b}$ and Leilei Yan ${ }^{1, c}$ \\ ${ }^{1}$ Center for Precision Engineering, Harbin Institute of Technology, Harbin 150001, China \\ acflying@hit.edu.cn, b2720130253@qq.com, c1562983704@qq.com
}

\begin{abstract}
Keywords: Ex-situ tracking technique, Morphological transformation, Triblock copolymer, AFM
\end{abstract}
Abstract. An ex-situ tracking technique for atomic force microscopy (AFM) was used for investigating repeatedly the imaging of some specific spaces on the whole substrate with a high relocation accuracy of tens of nanometers. As an example of the application of this technique, AFM ex-situ study of the morphology transition induced by solvent treatment in a block copolymer thin film has been carried out. By using single-wall carbon nanotube independent of microdomain patterns, even if the morphology of the block copolymer has been changed completely after a certain treatment, the previous imaged spot can be found easily.

\section{Introduction}

The microdomain structure in block copolymer thin films has recently attracted increasing attention both experimentally and theoretically [1-3]. These investigations are typically motivated either by the inherent interest in phase equilibrium in confined geometry or by the potential use of the well-ordered structures for lithographic applications. The atomic force microscopy (AFM) has been proven to be a useful tool for visualization of surface topography at the nanometer scale [4]. In comparison with other surface instruments such as electron microscopy and field ionic microscopy, AFM is much more widely used in the study of changes in the soft samples such as polymers, biological systems [5]. However, one of the main limitations with the AFM is that it is difficult or even impossible to relocate the same feature, object or the region of interest within a sample once the probe tip is lifted or the substrate is rotated or moved. A high precision relocation technique is needed for a great many applications.

This is delineated in a lot of research works of polymer transitions and biological processes, especially in research of the different morphology transitions of block copolymer thin films caused by either solvent or annealing treatment [6]. For example, in the ex-situ AFM study of morphology evolution, in order to observe and compare microdomains of block copolymers, it is necessary to zoom in an area at about $2 \times 2 \mu \mathrm{m}^{2}$ scale and precisely relocate the same spot later again after treating the sample. This is obviously a tedious and challenging task. On the other hand, if the morphology of the block copolymer has been changed completely after a certain treatment, some extra clues independent of microdomain patterns are needed. Otherwise there is no way to find the previous imaged spot.

In the pioneering work of P. Markiewicz and M.C. Goh, they utilized a suitable marker that could be mounted under the semi-transparent substrate as a routine relocation method in AFM. Although the method could sketchily locate the sample, it was time-consuming and its relocation precision was not very high. Hahm et al. used specially made substrate in order to perform the ex-situ AFM study of morphology evolution in a PS-b-PMMA block copolymer thin film under annealing conditions. However, it is not easy to make the specially substrate. In this paper, we report a relatively efficient and simple relocation technique achieved by utilizing single-wall carbon nanotube to perform an AFM ex-situ study of morphology development induced by solvent treatment in a block copolymer thin film. Comparing with the multi-wall carbon nanotube relocation method we reported in the previous research [7], single-wall carbon nanotube bring much less effect on the sample and the more minute morphology transition in block copolymer thin film can be traced by AFM. This relocating method not only has a high relocation precision, but also it is efficient and easy. It is believed that this kind of relocation method can be widely used in material science and molecular biology field besides polymer 


\section{Experiment}

Materials. The poly (styrene-ethylene/butylene-styrene) (SEBS) triblock copolymer coded as Kroton G-1650 was a commercial product of Shell Company. Its molecular weight, polydispersity and styrene content are $7.5 \times 10^{4}, 1.36$ and $27 \%$ respectively. Two solvents, xylene, a good solvent for both polystyrene (PS) and poly (ethylene/butylene) (PEB) blocks, and alcohol, are used in this work. Single-wall carbon nanotubes were purchased from Shenzhen Nanotech Port Company. A Branson ultrasonic cleaner (B3510E) was used for ultrasonic dispersion of single-wall carbon nanotubes. A Digital Instrument multimode SPM III atomic force microscopy (AFM) was used for viewing directly the cantilever and the backside surface features of the mica sheet.

Preparation of samples. The SEBS powders were first dissolved into xylene to prepare $0.3 \mathrm{wt} \%$ solution. Film was cast from $0.3 \mathrm{wt} \%$ solution onto a freshly cleaved mica substrate and then dried under ambient conditions. Some single-wall carbon nanotubes were dissolved into alcohol and

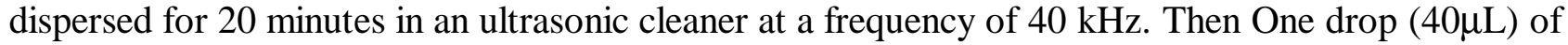
the solution was immediately cast (drop casting) onto SEBS film. The sample was imaged by AFM after its surface dried in ambient air. A piece of filter paper was put into the Petri dish to cover its bottom. The sample was put on the filter paper. After adding $500 \mu 1$ pure solvent xylene on the filter paper, the dish was sealed in a closed vessel and kept at room temperature. The sample was taken out at intervals of $0.25 \mathrm{~h}$ and measured after drying for few minutes in ambient air. In order to make up for loss of solvent, $200 \mu \mathrm{l}$ pure solvent xylene was added on the filter paper each time before the sample was put inside the Petri dish for solvent annealing again.

Relocation procedures. The used mica was cut into an inequilateral pentagon with a tip. In order to prevent the sample from being rotated, the orientation of the tip should be accordant every time. Fig. 1 shows that two sharp crossed scratches were made on the backside of the mica substrate beforehand as the mark for relocating a $5 \times 5 \mu \mathrm{m}^{2}$ large area. More similar marks can be made according to operator's will. Then the mica surface was cleaved and some $0.3 \mathrm{wt} \%$ solutions were dropped on it. The sample was dropped with single-wall carbon nanotubes solution after its surface dried in ambient air. The sample was imaged by AFM after its surface dried in ambient air again. Due to the transparency of the film and the mica substrate, the AFM tip was aligned from the film covered side with respect to the feature of the mark (an intersection of the two scratches) on the backside of the mica substrate by using the CCD and computer system.
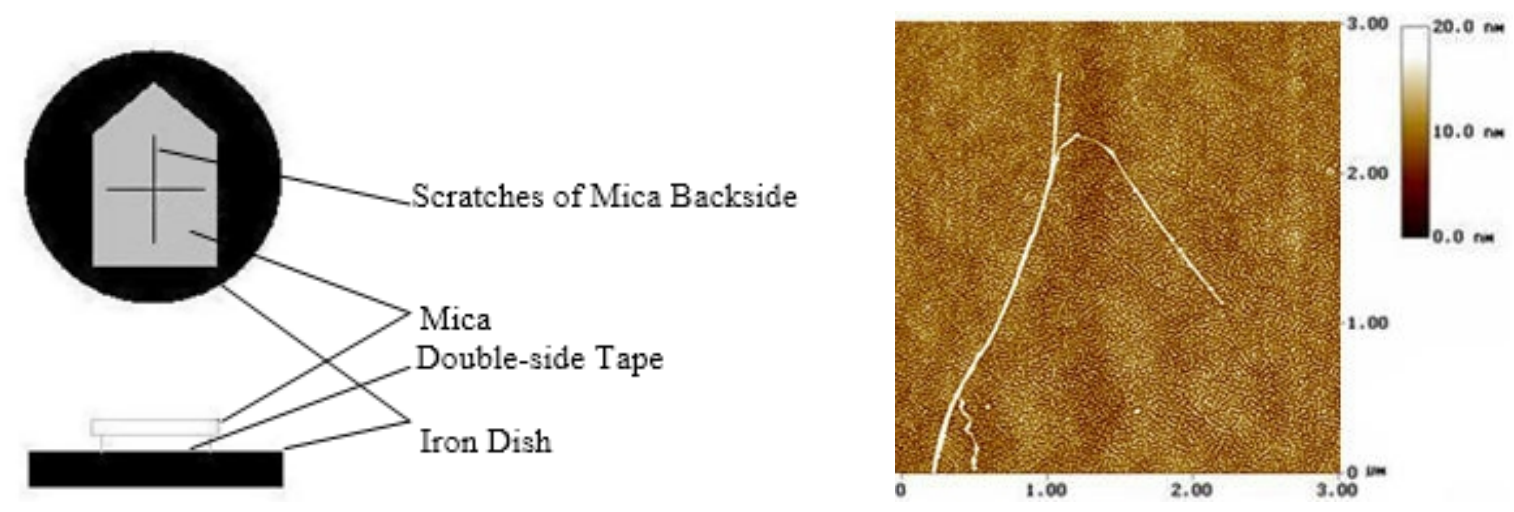

Fig. 1. A mica substrate that was marked by two sharp crossed scratches on the backside as a sample locator, was mounted on an iron sheet by a double-sided tape

Fig. 2. TappingMode AFM image of a large-scale scan to find the small area including single-wall carbon nanotubes

After the tip alignment, an single-wall carbon nanotube was observed on the film surface by scanning a $3 \times 3 \mu \mathrm{m}^{2}$ large area in TappingMode AFM (shown in Fig. 2). The single-wall carbon nanotube was used as the mark for relocating a smaller $1 \times 1 \mu \mathrm{m}^{2}$ area. 
AFM imaging. Digital Instrument multimode SPM III atomic force microscopy (AFM) was performed in Tapping Mode. The height and phase images were recorded simultaneously under a moderate tapping conditions (set-point ratio $r_{\mathrm{sp}} \approx 0.70$ ) with a free amplitude of $80 \pm 10 \mathrm{~nm}$, scan speed of $1.0 \mathrm{~Hz}$ and number of samples of $512 \times 512$. The operating frequency was readjusted after engaging the tip on the surface such that the operating frequency was kept on the low-frequency side of the resonance during the imaging. Commercial silicon probe tips with spring constant of about $50 \mathrm{Nm}^{-1}$ were used. All images were presented as only flattened raw data in order to filter the background slope.

\section{Results and discussion}

Fig. 3 shows a series of time lapse AFM height images recorded at the same spot during a sequential solvent annealing process. In the left area of all these images, the single-wall carbon nanotube that is responsible for relocating a $1 \times 1 \mu \mathrm{m}^{2}$ area can be seen. Microphase separation structure is clearly presented in these images. The higher domain (bright) corresponds to the polystyrene (PS) phase while the lower domain (dark) corresponds to polyethylene-butylene (PEB) phase.

Fig. $3 \mathrm{a}$ is the morphology of the as-cast film. The coexistence of a large number of PS cylinders, some with branches and a small number of PS spheres in the PEB matrix can be seen on the surface. This result is in good agreement with that obtained by TEM.

Fig. $3 \mathrm{~b}$ is the evolved morphology after $0.25 \mathrm{~h}$ solvent annealing of the as-cast film at ambient temperature. Fig. $3 \mathrm{c}$ is morphology of further solvent annealing for $0.25 \mathrm{~h}$ subsequently. By comparing the white circular area in Fig. 3a with that in Fig. $3 b$ and 3c, it can be seen that the minute change in morphology evolution process was captured.
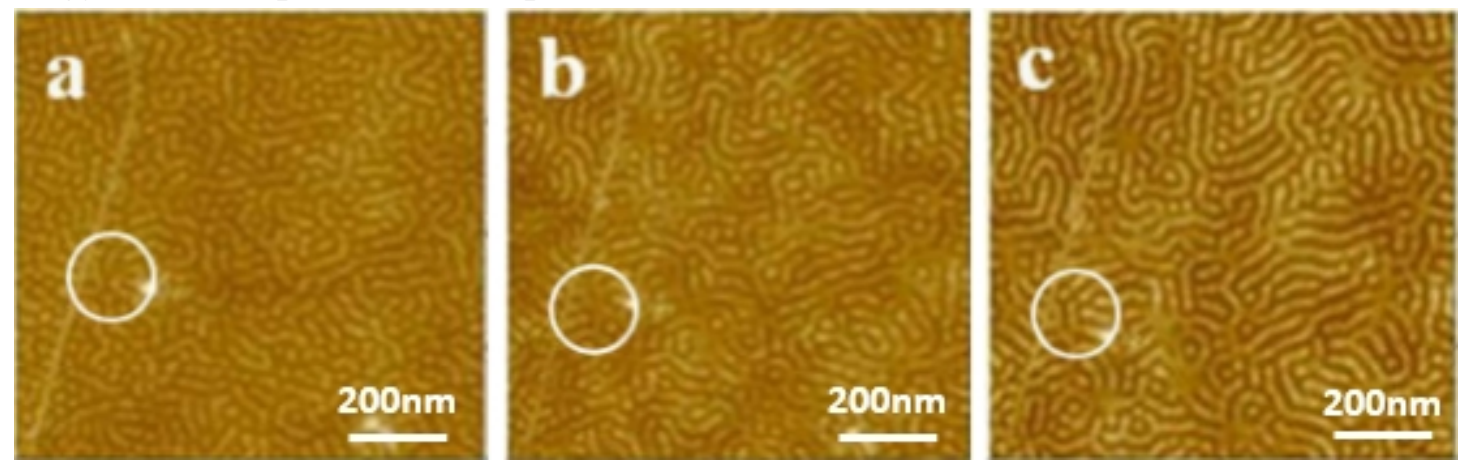

Fig. 3. A series of AFM images of block copolymer thin film recorded at the same spot before and after sequential solvent annealing treatments: (a) as-cast, (b) $0.25 \mathrm{~h}$ annealing, (c) $0.5 \mathrm{~h}$ annealing

By

carefully comparing Fig. 3a, 3b and 3c, it can be seen that there is a dramatic change of morphology that can be categorized into two aspects: First, the PS cylinders got more continuous by joining with each other. Second, partial single-wall carbon nanotube immerged in sample film in company with continuous fumigating and scanning. The above results show a distinct dependence of the thin film morphologies on the preparation conditions, in particular the solvent annealing. Using the technique, the preparation of films involves two competitive processes between phase-separation kinetics and vitrification due to the evaporation of the solvent. Because the vitrification prevents the phase separation from fully development, the morphology of thin films prepared under ambient conditions is usually in non-equilibrium (vitrified) state. Note that that the equilibrium structure formed in an ultrathin film where interfacial and confinement effects cannot be neglected does not necessarily coincide with the structure of a bulk piece of material.

Using the relocating procedures, in which the repeatedly positioning scale was reduced step by step, a more minor scanned scale relocation could be realized. A relocation precision of tens of nanometers can be reached by carefully controlling the movement of $x-y$ translation stage. Moreover, it is worth to mention that unlike previous AFM ex-situ study which depends on phase separation morphology clue 
to track the same spot, in this work, marks made on the film surface were used .The benefit of this method is that the required area will not be lost even if the morphology changes completely.

Comparing with the multi-wall carbon nanotube relocation method we reported in the previous research, single-wall carbon nanotube bring much less effect on the sample and the more minute morphology transition in block copolymer thin film can be traced by AFM. However, as the sample was scanned and imaged by TappingMode AFM repeatedly, single-wall carbon nanotube immerged in sample film. In order to avoid the single-wall carbon nanotube immerging in sample film entirely, less pure solvent xylene was added on the filter paper each time before the sample was put inside the Petri dish for solvent annealing again. Moveover, the tapping force was controlled to be lesser value.

This relocating method not only has a high relocation precision, but also it is efficient and easy. Therefore, it could be extensively used for AFM ex-situ study of polymer transitions and biological processes. We used a Nanoscope III system to relocate AFM samples, but the procedures outlined here could also be used for other different AFM only if it has the ability to view on the cantilever and sample backside features. It is noteworthy that AFM must be performed in Tapping Mode, because the sample with single-wall carbon nanotube will be destroyed if AFM is performed in Contact Mode.

\section{Conclusions}

A relatively efficient and simple relocation technique for TappingMode AFM, which takes advantage of single-wall carbon nanotube, is used for investigating repeatedly the imaging of some specific spaces on the whole substrate with a high relocation accuracy of tens of nanometers. As an example of the application of this technique, single-wall carbon nanotube was used to consistently relocate AFM tip and to perform an AFM ex-situ study of morphology transition in a triblock copolymer thin film induced by xylene solvent annealing. By using single-wall carbon nanotube independent of microdomain patterns, even if the morphology of the block copolymer has been changed completely after treatment, the previous imaged spot can be found easily. This relocating method not only has a high relocation precision, but also it is efficient and easy. It is believed that this kind of relocation method can be widely used in material science and molecular biology field besides polymer.

\section{Acknowledgements}

This work is supported by National Natural Science Foundation of China (No. 21573054 , 21327002 $\&$ 51401201), The Joint Funds Key Project of the National Natural Science Foundation of China (No.U1537214), The State Key Program of National Natural Science of China, (No. 51535003), Heilongjiang Postdoctoral Foundation of China (No.LRB07-140), and Programmed of Introducing Talents of Discipline to Universities (No. B07018).

\section{References}

[1] T. Thurn-Albrecht, J. Schotter, and G. A. Kastle: Science Vol. 290 (2000), p. 2126

[2] T.Hashimoto, J.Bodycomb, and Y.Funaki: Macromolecules Vol. 32 (1999), p. 2075

[3] M.D.Morariu, N.E.Voicu, and E.Schaffer: Nat Mater Vol. 2 (2003), p. 48

[4] J. P. Spatz, P. Eibeck, and S. Mossmer: Macromolecules Vol. 33 (2000), p. 150

[5] H. Elb, K. Fukunaga, and R. Stadler: Macromolecules Vol. 32 (1999), p. 1204

[6] M. A. van Dijk and R. van den Berg: Macromolecules Vol. 28 (1995), p. 6773

[7] Y. Z. Cao, Y. C. Liang, and S. Dong: Ultramicroscopy Vol. 103 (2005), p. 103 